\title{
Attentional modulation of binocular rivalry
}

\author{
Chris L. E. Paffen ${ }^{1}$ and David Alais ${ }^{2}$ \\ Department of Experimental Psychology, Helmholtz Institute Utrecht, Utrecht University, Utrecht, Netherlands \\ ${ }^{2}$ School of Psychology, University of Sydney, Sydney, NSW, Australia
}

\author{
Edited by: \\ Georgios A. Keliris, Max-Planck \\ Institute for Biological Cybernetics, \\ Germany

\section{Reviewed by:} \\ Ming Meng, Dartmouth College, USA \\ Teng Leng Ooi, Pennsylvania College \\ of Optometry at Salus University, \\ USA \\ *Correspondence: \\ Chris L. E. Paffen, Department of \\ Experimental Psychology, Helmholtz \\ Institute, Utrecht University, \\ Heidelberglaan 2, Utrecht 3584 CS, \\ Netherlands. \\ e-mail: c.l.e.paffen@uu.nl
}

Ever since Wheatstone initiated the scientific study of binocular rivalry, it has been debated whether the phenomenon is under attentional control. In recent years, the issue of attentional modulation of binocular rivalry has seen a revival. Here we review the classical studies as well as recent advances in the study of attentional modulation of binocular rivalry. We show that (1) voluntary control over binocular rivalry is possible, yet limited, (2) both endogenous and exogenous attention influence perceptual dominance during rivalry, (3) diverting attention from rival displays does not arrest perceptual alternations, and that (4) rival targets by themselves can also attract attention. From a theoretical perspective, we suggest that attention affects binocular rivalry by modulating the effective contrast of the images in competition. This contrast enhancing effect of top-down attention is counteracted by a response attenuating effect of neural adaptation at early levels of visual processing, which weakens the response to the dominant image. Moreover, we conclude that although frontal and parietal brain areas involved in both binocular rivalry and visual attention overlap, an adapting reciprocal inhibition arrangement at early visual cortex is sufficient to trigger switches in perceptual dominance independently of a higher-level "selection" mechanisms. Both of these processes are reciprocal and therefore self-balancing, with the consequence that complete attentional control over binocular rivalry can never be realized.

Keywords: binocular rivalry, visual attention

\section{HISTORICAL BACKGROUND}

When Wheatstone (1838) developed his mirror stereoscope in the first half of the nineteenth century, it was possible for the first time to independently control the presentation of images to the two eyes. In reporting his new device (Wheatstone, 1838), the main focus was on his impressive demonstration that presenting a matched image to each eye, and adding a small lateral displacement in opposite directions, produced a vivid sense of threedimensional depth. Wheatstone went on to discuss stereo-depth and its underlying geometry at length, however, with complete control over what was presented to each eye, Wheatstone's curiosity led him to try an obvious variation: what would happen if different images were presented to each eye? Toward the end of his paper, Wheatstone (1838) illustrates the kind of stereo-image he used to investigate this question. It consists of two different upper case letters, one for the left eye and one for the right, each letter presented within a matching fusion circle. He then describes what happens when such an image is viewed through a stereoscope, and in so doing provides the first systematic description of binocular rivalry:

"If $a$ and $b$ (fig. 25.) are each presented at the same time to a different eye, the common border will remain constant, while the letter within it will change alternately from that which would be perceived by the right eye alone to that which would be perceived by the left eye alone. At the moment of change the letter which has just been seen breaks into fragments, while fragments of the letter which is about to appear mingle with them, and are immediately after replaced by the entire letter. It does not appear to be in the power of the will to determine the appearance of either of the letters, but the duration of the appearance seems to depend on causes which are under our control: thus if the two pictures be equally illuminated, the alternations appear in general of equal duration; but if one picture be more illuminated than the other, that which is less so will be perceived during a shorter time. I have generally made this experiment with the apparatus, fig. 6 . When complex pictures are employed in the stereoscope, various parts of them alternate differently." (Wheatstone, 1838, p. 386, bold emphasis added)

Remarkably, Wheatstone's (1838) pioneering observations manage to capture all the main characteristics of binocular rivalry. He refers to the alternation of the monocular images, he mentioned the fragmented or "piecemeal" state that may occur during perceptual transitions, and he describes the changes in relative dominance associated with changes in stimulus strength. Importantly, he also claimed not to be able to determine which image dominates by an act of will. In so doing, Wheatstone (1838) made the first assertion in what was to be a long and vigorously contested debate: can binocular rivalry be controlled by voluntary attention? In Wheatstone's own time, this question engendered much discussion, with the great early scholars of perception voicing a variety of views on it (e.g., Hermann von Helmholtz, Ewald Hering, and William James). Indeed, arguments about the role of attention in binocular rivalry have continued to the present day and the issue remains central in contemporary rivalry research. One could argue that this question is central to the most recent major controversy 
in binocular rivalry: whether rivalry is eye-based or image-based (for a review of the controversy, see Blake and Logothetis, 2002), where an image-based view on binocular rivalry would allow more room for voluntary control.

As this historical background shows, the question of whether or not binocular rivalry is under voluntary (or attentional) control is as old as research into the fascinating phenomenon of rivalry itself. In this review, we will evaluate Wheatstone's (1838) claim that attention cannot determine rivalry dominance in light of research published since. In addition, we will review other studies which have sought to demonstrate a more moderate point, namely that attention can be used to modulate binocular rivalry, even if it cannot ultimately determine what is perceived when experiencing it. As well as reviewing the literature relating to rivalry and attention, we will also discuss recent findings suggesting that neural networks implicated in binocular rivalry and visual attention overlap. Overall, the review will focus as much as possible on studies of rivalry and attention. For more information about the many other aspects of binocular rivalry, the reader is referred to more general reviews (Blake and Logothetis, 2002; Alais and Blake, 2005; Tong et al., 2006; Blake and Wilson, 2011).

\section{VOLUNTARY CONTROL OVER BINOCULAR RIVALRY}

Among the first scholars to study binocular rivalry, it was Helmholtz (1925) who argued most forcefully that perception during rivalry was under volitional control. Indeed, Helmholtz claimed to have full control over what he perceived when viewing incompatible images, stating that "...I can retain the image of one pair or the other according to my fancy" (Helmholtz, 1925, pp. 497). On this view, where perceptual dominance is controlled by the observer's volition, the mechanism responsible for selecting the dominant image must be at a high-level of processing. If a lower-level mechanism were to determine perceptual dominance in rivalry, then it should not be under the observer's control. This led Helmholtz (1925) to conclude that binocular rivalry was indeed a high-level phenomenon, or as he labeled it: "a psychic act." In adopting this position, Helmholtz dismissed the "retinal view" taken by others such as Breese (1899) and Hering (1964) who emphasized the role of low-level factors in determining rivalry dominance.

Breese (1899) conducted his own experiments to test whether observers could control binocular rivalry. In his experiments, observers viewed a red and a green field presented separately to the eyes, and were instructed to try to hold one of them dominant. Breese (1899) found that observers could lengthen the dominance period of the nominated color field according to instruction, but that the average number of perceptual alternations did not change. He also noted that in trying to maintain dominance of one image, observers made vigorous eye movements. He suspected that eye movements exerted a substantial influence over which of the images was dominant and for how long, and he went on to conduct experiments on himself in which he kept his gaze tightly fixed while inspecting rival images. Under these conditions, he was not able to exert volitional control over rivalry dominance. This observation led Breese (1899) to conclude, as Hering (1964) had before him, that eye movements - rather than volitional control - were responsible for influencing perceptual dominance in binocular rivalry, and that the failure to control eye movements was the reason that Helmholtz was able to control perceptual dominance in rivalry.

In the ensuing decades, a number of other low-level and peripheral factors were studied for a possible confounding role in the apparent ability of attention to determine rivalry dominance. Simple fixational eye movements were further considered, as were the possible roles of eye blinks and other ocular motor acts such as the intrinsic eye muscle activity involved in pupillary constriction and accommodation. McDougall (1903) and George (1936), for example, paralyzed the eye muscles of a single eye and found that this decreased the amount of apparent voluntary control over rivalry dominance, but it did not eliminate it. Several other studies investigated the role of blinking in control over rivalry dominance (Washburn and Gillette, 1933; Bárány and Halldén, 1947; Meredith and Meredith, 1962), with each reporting that voluntary control over rivalry was still possible when accounting for blinking. Although these early studies addressing peripheral mechanisms and their possible confounding roles in volitional control over rivalry made strong claims, Lack (1978) surveyed the literature and noted that all these studies were poorly conducted. For example, McDougall (1903) and George (1936) used only one observer in their studies, and Meredith and Meredith (1962) did not actually measure blink rates in their investigation. Clearly, a properly controlled reinvestigation of these peripheral factors was needed.

In his dissertation "Selective attention and the control over binocular rivalry," Lack (1978) presents several carefully controlled experiments designed to address the limitations of these preceding studies of peripheral ocular factors in rivalry, whose claims had been lingering for almost a century. In a systematic series of experiments, he was able to exclude a role for eye movements, blinking, accommodation, and pupillary activity as potential confounds in the apparent ability to voluntarily control binocular rivalry. With these peripheral factors excluded, Lack then returned to the central question: can observers attentionally control binocular rivalry? Lack's (1978) studies first established that unpracticed observers were able to exert a modest degree of attentional control over binocular rivalry, and he went on to show that the extent of voluntary control could be increased with practice. Thus, Lack's (1978) important study demonstrated two significant points. First, the claim against attention's control over rivalry, which was based on uncontrolled peripheral factors, could be ruled out. Second, with peripheral factors controlled, Lack (1978) showed that perceptual dominance in binocular rivalry could be significantly influenced by an act of selective attention (we will return to this point later). In the history of binocular rivalry, Lack's (1978) study was a turning point that paved the way for a return to an examination of the role of attention in rivalry.

In recent years, studies by Meng and Tong (2004) and van Ee et al. (2005) have revived the issue of voluntary control and reach a similar conclusion to that of Lack (1978): control over binocular rivalry is possible. Meng and Tong (2004) compared the extent to which attentional control was possible for binocular rivalry and for the Necker cube. They instructed observers to try to hold one image dominant (or one perspective, in the case of the Necker cube) at the cost of the other, and compared this to a neutral baseline. They observed that a degree of attentional 
control was possible for both stimuli, but that there was less control over perception during binocular rivalry than for the Necker cube. However, when observers followed a different instruction to try to attentionally modulate the rate of perceptual alternation in the displays - the effect of attention on alternation dynamics was considerably greater, and comparable control was observed for rivalry and the Necker cube. Thus, although attentional control over binocular rivalry was possible, the authors concluded that rivalry involved a more automatic, stimulus-driven form of perceptual bistability than did the Necker cube.

A paper by van Ee et al. (2005) also looked at attentional modulation of alternation dynamics in several bistable displays, comparing binocular rivalry, the Necker cube, and a bistable stereo-slant stimulus. Similar to Meng and Tong's (2004) observation, van Ee et al. (2005) also found that attentional control over binocular rivalry dynamics was less than was possible for other perceptually bistable displays. In related papers (van Dam and van Ee, 2006a,b), the role of eye movements in voluntary control over bistable stimuli was re-investigated, returning to the possible contribution of peripheral mechanisms to voluntary control. These studies analyzed the role of eye movements and found a positive correlation between saccades and perceptual alternations during binocular rivalry, implying that eye movements can be used actively to instigate a perceptual alternation. Interestingly, however, these authors found that the role of saccades was not different in voluntary control conditions. Thus, when trying to control perception during rivalry, observers did not use eye-movement strategies that were any different to those employed during passive viewing. This conclusion agrees with Lack's (1978) investigation nearly 30 years earlier and confirms that voluntary control over binocular rivalry is indeed possible and cannot be explained by eye movements.

Interestingly, a recent study showed that voluntary control over binocular rivalry can be increased when accompanied by congruent auditory information (van Ee et al., 2009). Observers viewed a rival display consisting of a looming and a rotating pattern. When instructed to try to hold the looming percept dominant, attentional control was greater when a correlated looming sound accompanied the visual stimulus. This result shows a cross-modal influence on binocular rivalry and therefore broadens the search for the attentional mechanisms underlying voluntary control over binocular rivalry. Moreover, this cross-modal effect on binocular rivalry was only observed when the stimuli were attended: the mere presence an auditory signal correlated with the visual stimulus that observers were instructed to maintain in dominance did not help them in their task. The auditory signal had to be actively attended if the sound signal were to help to maintain the looming percept dominant in binocular rivalry. The correlation between the auditory signal and the visual stimulus to be attended was also critical, as a looming sound with a different rate to the looming visual stimulus was ineffective at enhancing perceptual control. In a couple of interesting extensions of this work, the authors also report that a correlated tactile stimulus is equally effective as the correlated auditory signal at facilitating attentional control over visual rivalry, and that a combination of auditory and tactile stimuli is even more effective. In addition, control over a bistable auditory stimulus was found to be enhanced by adding a correlated visual stimulus.

\section{ATTENTIONAL MODULATION OF BINOCULAR RIVALRY}

The earliest debate surrounding the role of attention in binocular rivalry was primarily concerned with whether perception during rivalry could be controlled entirely by an act of will. The notion that attention and rivalry may be closely linked has a neat appeal. There is a clear analogy between attentional selection among competing objects and perceptual selection in rivalry between competing images. Although appealing, this notion - at least in its strongest form - is easily overturned by empirical experience as selecting an image for perceptual dominance is only partly under the control of an observer. This led to the role of attention in rivalry being ignored for a long period. Lack's (1978) careful experimenting on the topic, however, opened the door for a weaker form of the attention hypothesis by showing clearly that attention is at least a factor at work in binocular rivalry, even if it is not the primary or causative one. Not surprisingly, therefore, the last decade or so has seen a number of studies published that have revisited the issue of attention and rivalry and asked a more subtle question: how can the various forms of attention modulate (if not totally control) binocular rivalry?

A fundamental distinction is drawn in the attention literature between voluntary or endogenous attention and involuntary or exogenous attention (see Bundesen and Habekost, 2008; Wright and Ward, 2008). Both are acts of attentional selection, but endogenous attention is a voluntary choice to focus on an object, location, or feature (an act of will, or as Helmholtz called it: "immediate attention") whereas exogenous attention occurs when a stimulus onset or stimulus change captures attention and is automatically selected. This is an involuntary form of attention (or "mediate attention" in Helmholtz's terms). The first paper (Ooi and He, 1999) among the recent flurry of studies examining attention and rivalry investigated how both forms of attention - voluntary and involuntary - affect binocular rivalry. Ooi and He (1999) first investigated the ability of voluntary attention to sustain dominance of a selected rival target. To do this, they exploited a well-known effect that a transient change in the suppressed eye's stimulus is an effective way to trigger a perceptual switch to that eye (Grindley and Townsend, 1965; Walker and Powell, 1979). Ooi and $\mathrm{He}$ (1999) instructed observers to attend to one of four targets presented to the dominant eye, and a transient perturbation was made to the stimulus in the suppressed eye. They observed that when the transient was located at the location corresponding to the attended target in the dominant eye, dominance of that target was terminated less often than when the transient occurred at the location of one of the three unattended targets in the dominant eye. This result shows that voluntarily attending to a dominant image will help maintain the "selected" image in a state of perceptual dominance.

Ooi and He (1999) also investigated whether involuntary attention directed to a suppressed stimulus could break its suppression and cause the suppressed stimulus to become dominant. They tested this using a monocular pop-out cue (a pair of parallel lines) that flanked the location of one rival target in a circular array of six rivaling targets. A cue was added around all six targets (thereby controlling for stimulus onset) but five cues were horizontal line pairs and the critical sixth cue was a vertical pair and thereby popped out of the display as an effective exogenous cue. 
The authors found that this salient monocular cue significantly enhanced the tendency of the cued target to achieve dominance, relative to the non-cued targets. This finding demonstrated an effect of involuntary attention on the suppressed image in that the suppressed image was more likely to return to dominance when attention was drawn to it automatically by a salient monocular pop-out cue. Together with their manipulation of endogenous attention, this study provided some of the first empirical evidence that both endogenous and exogenous attention can modulate the neural processes underlying binocular rivalry.

In a recent study by Paffen and Van der Stigchel (2010) it was shown that involuntary attention can also initiate a perceptual alternation: in their experiments, rival targets were presented both to the left and right of fixation. Observers were instructed to press either of two buttons corresponding to the two rival locations whenever an alternation was perceived at these locations. At random intervals, an exogenous cue surrounding the rival targets was presented at one of the two locations. The results showed that alternations occurred earlier as well as more frequently at the location where the cue was presented. These results suggest that the occurrence of perceptual alternations is related to the spatio-temporal properties of visual attention: moving attention to a spatial location increases the chance of perceiving a perceptual alternation at that location.

\section{DOES RIVALRY OCCUR IN THE ABSENCE OF ATTENTION?}

From the studies discussed so far, it is clear that attentional selection can be used to modulate binocular rivalry. Selecting one image from a pair of rivaling images, whether by exogenous or endogenous attentional selection, will suffice to reveal this. Endogenously attending to the dominant image will extend its dominance duration, and exogenously cueing attention to the rival images will increase the likelihood of a perceptual switch (Ooi and He, 1999; Paffen and Van der Stigchel, 2010). But what if attention is withdrawn from the rival images? This question was addressed in an influential early study by Ooi and He (1999). In the final experiment of that paper, observers were briefly presented with a pair of rival images, one of which was preceded by a surrounding cue. Observers had two tasks to do. The first was to report which of the two rival images became dominant first. The critical manipulation, however, involved presenting a Vernier target at a different spatial location and having the observer perform a second task: was the Vernier stimulus offset to the left or to the right? A preceding experiment had shown that cueing an image usually caused it to dominate first. The results of the dual-task experiment showed that this cueing effect was reduced: the cued image became dominant less often in the divided attention condition, compared to a focused condition with no Vernier task. This result nicely shows that dividing attention between two tasks weakens its ability to select a given stimulus at rivalry onset.

Ooi and He's (1999) experiment concerned dominance at rivalry onset. What about ongoing rivalry alternations? One of the hallmarks of binocular rivalry is that constant visual input leads to spontaneous and ongoing changes in perceptual dominance. What happens to these alternations when attention is divided? If withdrawing attention from the rivalry stimuli to a demanding competing task were to eliminate perceptual alternations, it would imply that attention is necessary for binocular rivalry to occur. This question was addressed by Paffen et al. (2006) in a dual-task paradigm. They had observers continually track their rivalry alternations while also performing a secondary task at a different spatial location. Surrounding the rivalry stimuli was an annulus containing incoherent random-dot motion. Occasional bursts of weakly coherent motion intermittently replaced the incoherent motion, and observers had to detect when these weak motion bursts occurred. With this dual-task paradigm, Paffen et al. (2006) found that the rate of rivalry alternations decreased when observers had to detect the motion bursts, compared to when the motion was ignored. Thus, withdrawing attentional resources from the rivalry stimuli slowed the rate at which rivalry alternations occurred, but did not abolish them. As attentional resources might still have been deployed to the rival images while detecting motion bursts, the authors went on to increase the difficulty of the motion detection task. Even when the motion detection task was difficult ( $\mathrm{d}^{\prime}$ were around 1 ), rival alternations were far from arrested: alternation rates were still around 0.35 alternations per second. Although these results show that rival alternations still occur when attentional resources are withdrawn, it is clear that attentional resources affect the rate at which rival alternations occur: when attentional resources are withdrawn, rival alternations become slower. A similar result was recently reported by Paffen and Hooge (2011). In this study, observers reported perceptual alternations in multiple rival images. The results showed that the number of alternations reported per rival pair went down when the number of rival images was increased. Their results imply that distributing spatial attention also slows the speed of binocular rivalry.

Notably, withdrawing attention does not only slow alternations during binocular rivalry, but affects other instances of bistable perception as well (e.g., Reisberg and O'Shaughnessy, 1984; Pastukhov and Braun, 2007). For example, Pastukhov and Braun (2007) performed experiments in which attention was withdrawn from a bistable plaid stimulus. Even when attentional deployment to the plaid was minimized, perceptual alternations still occurred. Interestingly, when attentional resources are deployed in another modality, rival alternations also become slower: Alais et al. (2010b) recently showed that attending to auditory signals also slows alternations in a visual rival display. In addition, it was reported that withdrawing attention had a bigger effect when rivalry was instigated between images of a house and a face, than when instigated between two gratings. This result corroborates with those reported by van Ee et al. (2005): in that study, observers had greater (voluntary) control when rivalry occurred between images of a house and a face then when between sinewave gratings. This seems to imply that the influence of attention on rival images increases as these images are analyzed higher up the visual processing stream.

To our knowledge, there is one neuro-imaging study investigating the withdrawal of attention on binocular rivalry. For this, Lee et al. (2007) used the phenomenon of traveling waves that occurs when rival images are of considerable size: a transition from one percept to the next often involves a local breakthrough of the suppressed image, followed by a traveling wave of emergent perceptual dominance of the other parts of the suppressed image (Wilson et al., 2001). When attention was directed to the rival 
images, traveling waves of activity in V1, V2, and V3 correlated both temporally (i.e., the speed of the waves in perception and neural tissue) and spatially (the location of the waves in the visual field and retinotopically in the neural tissue) with perceived traveling waves. When attention was diverted to monitoring letters at the center of the display activity in V1 still correlated with perception of the dominance wave, in V2 and V3, however, the correlation was abolished. Thus, when attention was diverted, rivalry-related activity was present in V1, but not in later areas.

\section{DOES BINOCULAR RIVALRY POP-OUT?}

To this point, we have demonstrated a number of ways by which attention can modulate binocular rivalry. A converse question can also be asked: is a rivaling stimulus able to automatically attract attention? In other words, is binocular rivalry (or more specifically, "interocular conflict") a pop-out attribute? The first investigation of this question (Wolfe and Franzel, 1988) involved a series of experiments in which observers searched for targets defined by interocular conflict among an array of distractors that were interocularly matched. The stimuli were square-wave grating patches, with the distractors being interocularly matched in orientation and the target being orthogonally oriented gratings. By measuring search times for such targets among various numbers of distractors, search efficiencies can be calculated (i.e., search time per item). The results showed that search for a target defined by interocular conflict was not parallel (or in contemporary terms, was not "efficient"), nor was it much faster than the converse relationship (searching for a target of fused orientations among rival distractors). From this investigation, Wolfe and Franzel (1988) concluded that targets defined by binocular rivalry do not "pop-out."

In a recent study, however, Paffen et al. (2011), showed that search for targets defined by interocular conflict could lead to a "near efficient" search (corresponding to search slopes of about $15 \mathrm{~ms}$ per item), depending on the stimulus conditions. These authors found that slow search times occurred when high-contrast stimuli were used, but that search times were faster and more efficient for lower contrasts. While this explains the discrepancy with Wolfe and Franzel's (1988) findings (they used a high-contrast display of white gratings on a black background), it does seem counterintuitive: lowering the contrast of a search target generally attenuates search performance (Pashler et al., 2004). However, when dissimilar images are presented dichoptically, it takes a while for binocular rivalry to occur: during the first $150 \mathrm{~ms}$ or so, images do not rival but instead undergo a "false fusion" (Wolfe, 1983). This is not normal fusion (as the monocular images are not matched) and observers can easily discriminate between fused images and images that are fused optically (Georgeson and Meese, 1997). Furthermore, it has been shown that the duration of the false fusion period (or "proto-rivalry," as it was labeled by Solomon et al., 2006) increases as the contrast of rival images decreases (Liu et al., 1992). Paffen et al. (2011) reasoned that this relationship was the cause of the higher search efficiency observed in low contrast displays. Indeed, when Paffen et al. (2011) presented the search displays for just $150 \mathrm{~ms}$ (thereby optimizing abnormal fusion), observers could easily detect the search target defined by interocular conflict.

\section{HOW DO ATTENTION AND RIVALRY INTERACT?}

As has been discussed above, the debate about the relationship between binocular rivalry and attention has been present since research into rivalry first began. For Helmholtz (1925), binocular rivalry was a psychic act: keeping an image dominant in perception during binocular rivalry was an act of both immediate (voluntary) and mediate (involuntary) attention. If it seemed keeping an image dominant in perception by mere voluntary attention was difficult, it was because the image would eventually cease to be new, and voluntary control would become more difficult. Helmholtz thought this tendency could be counteracted by keeping an image interesting, for example, by counting the number of lines in the display. The parallel with visual attention is obvious: selection of visual information can occur voluntarily, but can also occur when aspects of the stimulus can attract attention automatically. More recently, after reviewing several commonalities between attention and binocular rivalry, Leopold and Logothetis (1999) concluded that "mechanisms of selective attention and multistability might be closely related" (Leopold and Logothetis, 1999). This conclusion was based on two general observations. First, both visual attention and binocular rivalry involve competition in which some information is selected at the expense of other information. Second, neuroanatomical networks of visual attention and binocular rivalry show considerable overlap. We will discuss these two observations below.

The first observation, that visual attention and binocular rivalry are both acts of selecting information, was used in a study by Mitchell et al. (2004). In their experiments, observers binocularly viewed two counter-rotating fields of rotating dots that were superimposed in transparent motion. Attention was then cued to one of the surfaces by a brief translational motion pulse after which the viewing conditions quickly changed to dichoptic, with each surface presented to a separate eye to trigger binocular rivalry. The authors observed that the surface that was cued prior to the initiation of dichoptic viewing tended strongly to be the dominant image in the first period of rivalry. Based on these results, the authors concluded that "attention and rivalry rely on shared object-based selection mechanisms" and that both "engage common competitive mechanisms" (Mitchell et al., 2004). A related result has earlier been published by Ooi and $\mathrm{He}$ (1999), who showed that pre-cuing the location of one rival target among an array of six rival targets raised the likelihood that it would become dominant at rivalry onset. While both these two studies used exogenous cues, Chong and Blake (2006) went on to study the effect of an endogenous cue on initial phase of rivalry dominance. Observers were presented with binocularly viewed plaids whose component gratings underwent independent changes in orientation and spatial frequency. Observers were instructed to direct their attention to one of the component gratings. Similar to Mitchell et al.'s (2004) procedure, each of the gratings was then presented separately to the eyes and the same result was reported: initial dominance tended to favor the endogenously attended grating.

As the above discussion shows that attention and binocular rivalry do interact, the next question is how they interact. A few studies suggest that attention affects binocular rivalry by affecting the effective contrast of the rivaling images (Chong et al., 2005; 
Chong and Blake, 2006; Paffen et al., 2006; Paffen and Hooge, 2011). This notion is motivated by two observations. First, changing the contrast of rival images has a profound impact on the temporal dynamics of rivalry: lowering the contrast of both images will lengthen the time that each image is dominant in perception (e.g., Levelt, 1965; Brascamp et al., 2006). Second, attending a stimulus is known to increase its perceived contrast (Carrasco et al., 2004), a finding that parallels the finding that attending a stimulus (contrasted with disattending the stimulus) affects neural responses in a manner similar to increasing the contrast of that stimulus (Reynolds and Chelazzi, 2004). Both Chong et al. (2005) and Paffen et al. (2006) used these two observations and reasoned that one influence of attending to the rival stimuli could be to increase their effective contrast. Chong et al. (2005) had observers track spatial frequency changes of one of two rival targets, or increased the contrast of one of the targets as soon as it became dominant. These authors reported that both attending to the grating and increasing its contrast were effective at increasing the perceptual dominance of that grating.

The relationship between attention and contrast in binocular rivalry was also examined in Paffen et al.'s (2006) study. It has already been noted above that observers tracked rivalry alternations in a central stimulus while at the same time detecting brief motion bursts in an annulus surrounding the rival targets. This attentional condition was compared with a passive viewing condition, and both were done at four levels of contrast. The results showed that diverting attention to the motion detection task had a quantitatively similar effect on slowing the alternation rate as halving the contrast of the rival targets. Together, these results and those of Chong et al. (2005), with the tight relationship between stimulus contrast and rivalry alternation rate, show that attention's influence on binocular rivalry can be modeled as a change in effective contrast. When rival targets are attended, the effective contrast of the targets increases, leading to an increase in alternation rate equivalent to that produced by increasing stimulus contrast by roughly a factor of two (Paffen et al., 2006).

The observation that attention affects rivalry by boosting its effective contrast predicts that attention and contrast should influence rivalry in the same way. Interestingly, Levelt (1965) noticed that changing the contrast of only one rival image actually affected the dominance duration of the other image, leaving its own dominance unaffected. This famous finding (formalized into Levelt's 2nd proposition) leads to the counterintuitive prediction that attending to one rival image will affect the dominance of the other image, and not the attended one. Several studies have addressed this issue. In the attention condition of Chong et al.'s (2005) study, the mean dominance duration of the attended grating was increased by as much as $50 \%$, but the mean dominance duration of the unattended grating was no different from what was observed in the passive viewing condition. This result shows that attention only exerts its influence on the stimulus that is perceptually dominant, and not on the suppressed stimulus, thereby violating what would be expected from Levelt's 2nd proposition. However, as Chong et al. (2005) remark, this is not surprising because one can only attend to an image that it is perceptually present and available for selection; it is impossible to select an image that cannot be seen. Their results square with those on voluntary control mentioned above, where voluntary control generally increases the time the attended image is perceived. In contrast to these findings, Hancock and Andrews (2007) reported that attending one of two rival gratings decreased the mean dominance duration of the unattended grating, leaving that of the attended grating unaffected. The latter result is in correspondence with Levelt's 2nd proposition. At present it is unclear what exactly explains the discrepancy between the findings of Chong et al. (2005) and those of Hancock and Andrews (2007) but there is clearly more to discover about the role of attention on rivalry dominance durations.

The second important point that Leopold and Logothetis (1999) made was that a number of the brain areas involved in attention are also implicated in binocular rivalry. At the core of this is the observation that both attention and binocular rivalry involve a distributed fronto-parieto-occipito network that is crucial in attentional selection and mediating perceptual alternations during binocular rivalry. In the case of visual attention, the existence of such a network is relatively undisputed: many studies have shown fronto-parietal areas to be crucial in exerting topdown control over visual perception (Posner and Dehaene, 1994; Desimone and Duncan, 1995; Kastner and Ungerleider, 2000; Corbetta and Shulman, 2002; Bisley, 2011). As an example, Zanto et al. (2011) recently targeted frontal areas with rTMS and found that this lead to diminished top-down modulation of visual processing in posterior (visual) areas. In binocular rivalry, perception-related activity has been found as early as the lateral geniculate nucleus (LGN: Haynes et al., 2005; Wunderlich et al., 2005), primary visual cortex: (Polonsky et al., 2000; Tong and Engel, 2001; Lee et al., 2005) and later processing areas such as fusiform face area (FFA) and the parahippocampal place area (PPA: Tong et al., 1998). Interestingly, one of the first imaging studies of binocular rivalry found transient activation related to perceptual alternations in parietal and prefrontal areas (Lumer et al., 1998). In this study, BOLD activity was contrasted between observers viewing rival displays and displays in which rivalry was mimicked (images were presented in alternation on the display). The results showed that right frontoparietal regions showed greater activation during rival alternations than during simulated rivalry. The authors noted that these brain regions were also involved in spatial attention and suggested that both visual attention and binocular rivalry "may there for call upon a common neural machinery in fronto-parietal cortex, involved in the selection of neuronal events leading to visual awareness" (Lumer et al., 1998).

Subsequent to Lumer et al.'s (1998) study, several later studies also found this fronto-parietal network to be implicated in binocular rivalry (Lumer and Rees, 1999; Srinivasan et al., 1999; Miller et al., 2000; Cosmelli et al., 2004; Sterzer and Rees, 2008). The involvement of frontal and parietal regions in binocular rivalry, therefore, is not disputed. The question remains, however, whether perceptual alternations during rivalry arise in these areas and mediate earlier processing via feedback (as suggested, for example, by Leopold and Logothetis, 1999), or whether perceptual alternations arise at an earlier level of visual processing and then feed forward to fronto-parietal areas. The feedback possibility would more closely correspond to the notion of frontal areas exerting top-down control over visual perception, as in the case of selection by attention. The feedforward possibility would correspond 
more with a bottom-up component to perceptual alternations during rivalry with the competition between the monocular inputs resolved at early levels, where subsequent percept-related activity can be found higher up the processing stream. There is some evidence for the feedback possibility (Sterzer and Kleinschmidt, 2007; Britz et al., 2009), where frontal (Sterzer and Kleinschmidt, 2007) and parietal (Britz et al., 2009) activity precedes occipital activity associated with perceptual alternations. Importantly however, these results were obtained by using a complex Necker cube (Britz et al., 2009) and apparent motion (Sterzer and Kleinschmidt, 2007) stimuli which, although clearly bistable, lack the interocular mismatch that triggers rivalry. This difference might explain the apparent difference with a study by Kamphuisen et al. (2008), who used binocular rivalry stimuli and observed that while parietal and frontal activation were involved in mediating perceptual alternations, a phase analysis of this activity showed it was the result of occipital sources.

Recently, is has become evident that regions of parietal cortex are particularly important in mediating perceptual alternations. Carmel et al. (2010) applied TMS over right superior parietal cortex (SPL) and found that this shortened dominance durations during binocular rivalry. In addition, Kanai et al. (2010) found that cortical thickness of bilateral superior parietal cortex was negatively correlated with the speed of perceptual alternations triggered by a structure-from-motion stimulus. Interestingly, these authors went on to apply continuous theta-burst stimulation (cTBS) over right and left SPL and found that applying this kind of TMS increased percept durations. The apparent contradictions between the two studies was resolved in another study by Kanai et al. (2011). By applying a ROI analysis based on the relation between percept durations and cortical thickness reported by Kanai et al. (2010), different sub-regions of SPL were targeted with offline TMS. Disrupting right anterior SPL was found to shorten percept duration, while disrupting right posterior SPL increased percept durations. Importantly, these results apply both to perceptual alternations caused by binocular rivalry stimuli and a structure-from-motion stimulus, implicating that these areas are similarly involved in both kinds of bistability. Although this study resolved the conflicting results of Carmel et al. (2010) on the one hand and Kanai et al. (2010) on the other, they are in conflict with another study showing that online TMS over anterior SPL increases percept durations during binocular rivalry (Zaretskaya et al., 2010). The reason for this discrepancy is not clear, although one notable difference between the studies is that Zaretskaya et al. (2010) used online TMS, whereas Kanai et al. (2011) used offline TMS. This difference may be critical, as it is possible that the region targeted by offline TMS spreads during the offline period to affect non-targeted regions during testing. More research will be needed to resolve this discrepancy but for the moment these TMS studies show that parietal cortex, a crucial structure in (spatial) attention (e.g., Corbetta and Shulman, 2002), also has a critical role in determining binocular rivalry dynamics. It is less clear what these studies imply regarding feedforward versus feedback initiation of perceptual alternations because apart from affecting processing in parietal areas, TMS likely also affects the feedback/feedforward interplay between parietal and other processing areas at both lower and higher stages.
A specific involvement of frontal areas in bistable perception has recently been implicated by Windmann et al. (2006). This study used patients with prefrontal lesions who were given one of three instructions; to hold one of the two bistable percepts, to look passively at the stimulus, or to try to speed up the rate of alternations. Interestingly, the ability to hold a percept was not different between patients and controls, however, patients were less able to speed up the rate of perceptual alternations by will. Although binocular rivalry was not used in this study, it suggests that prefrontal cortex serves a specific role in attentional control over bistable stimuli. As the authors remark, damage to prefrontal cortex might have hampered the ability to intentionally switch between two images, leaving the ability to hold a percept unaffected.

\section{CONCLUDING REMARKS}

We noted in the introduction that it was Wheatstone (1838) who first posed the question of whether binocular rivalry can be controlled by will. More than 170 years later, and with the benefit of techniques and methodologies that were not even conceivable when he published his pioneering observations in 1838, we can now answer his question: voluntary control over binocular rivalry is possible, yet limited. Like many highly polarized debates, the answer has turned out to be a mixture of both positions. Carefully controlled studies have clearly shown that observers can use attention to modulate their perception during binocular rivalry, either lengthening the dominant percept's duration or changing the overall alternation rate (Lack, 1978; Meng and Tong, 2004; van Ee et al., 2005). Complementing this is the wealth of data showing that binocular rivalry depends strongly on low-level visual attributes such as orientation, spatial frequency, and contrast (Blake, 1989).

The conclusion that binocular rivalry can be partially controlled by voluntary attention is suggestive of an interplay between top-down and bottom-up factors. The fact that observers cannot voluntary take full control over their perception in rivalry suggests that bottom-up factors are important. It has long been theorized that binocular rivalry is initiated early in cortical processing, at a stage where monocular inputs are first combined, and that reciprocal inhibition exists between the monocular neural populations (Blake, 1989). The balance between left- and right-eye neurons varies over time because of adaptation, changing the balance, and causing perceptual switches (Alais et al., 2010a). Within this framework, the image that happens to be dominant at a particular point in time is available to be attentionally selected by top-down processes. As is now well established, this will boost the neural response to the selected image (Reynolds and Chelazzi, 2004) and raise its effective contrast, causing it to dominate more. However, this process cannot continue indefinitely as there is also adaptation taking place that will weaken the response to the dominant image and inevitably lead to a switch in perceptual state. Thus, just as there is a reciprocal relationship between the left- and righteye's responses, there is also a reciprocity between, on one hand, the response-boosting effect on the early neurons from top-down selection (Reynolds and Chelazzi, 2004), and on the other hand, the response decrement effect due to neural adaptation in these early neurons. Because of this reciprocity, an observer's attempt 
to attentionally control their perception in rivalry is inevitably limited.

While the primary role proposed for early interocular interactions in binocular rivalry has been contrasted with results implying that binocular rivalry involves later processing stages (Kovács et al., 1996; Logothetis et al., 1996), these results can be integrated within the contemporary view that binocular rivalry involves a multitude of visual stages from early to late processing (Ooi and $\mathrm{He}$, 1999; Blake and Logothetis, 2002; Wilson, 2003; Blake and Wilson, 2011). This "distributed network" view also provides the neural framework within which the top-down/bottom-up interplay that we are proposing can take place. From the literature reviewed above it is obvious that neuro-anatomical networks involved in attentional selection and binocular rivalry overlap. There is also evidence that other networks maybe involved in interocular suppression. A series of fMRI studies by He and colleagues (Fang and He, 2005; Jiang and He, 2006) showed that for some classes of stimuli, a cortical response was still elicited even when they were perceptually suppressed. This was found in dorsal areas for visual objects such as tools, and in FFA, and STS for faces, especially fearful faces. Further, it has been found that when erotic images are suppressed they are still able to influence the spatial allocation of attention (Jiang et al., 2006). Although these studies used continuous flash suppression (Tsuchiya and Koch, 2005) rather than conventional binocular rivalry, they do suggest interesting possibilities relevant to this review. One is that visual information could arrive in extrastriate visual cortex through subcortical pathways and bypass early suppression (Weiskrantz, 1997; Morris et al., 1999). A second possibility is that suppression is an attenuation process that leaves signals weakened but still able to activate subsequent areas, albeit without awareness. Broadly, these findings are consistent with other recent evidence suggesting a multistage rivalry process (Nguyen et al., 2003; Alais and Melcher, 2007) and that dorsal and ventral rivalry-processes may be independent (Alais and Parker, 2006).

Although it is tempting to conclude that the fronto-parietal areas involved in attentional selection (Duncan, 2001; Miller and Cohen, 2001) must also be involved in selecting and maintaining the dominant image during binocular rivalry, it is not clear that this conclusion follows. Although a role for these areas in selection may

\section{REFERENCES}

Alais, D., and Blake, R. (2005). Binocular Rivalry. Cambridge: MIT Press.

Alais, D., Cass, J., O’Shea, R., and Blake, R. (2010a). Visual sensitivity underlying changes in visual consciousness. Curr. Biol. 20, 1362-1367.

Alais, D., van Boxtel, J. J., Parker, A., and van Ee, R. (2010b). Attending to auditory signals slows visual alternations in binocular rivalry. Vision Res. 50, 929-935.

Alais, D., and Melcher, D. (2007). Strength and coherence of binocular rivalry depends on shared stimulus complexity. Vision Res. 47, 269-279.

be evident in some circumstances, the involvement of these areas does not appear to be strictly necessary to select a "winner" from the competing rivalry stimuli. That is, it is clear that an adapting reciprocal inhibition model of rivalry can also explain perceptual switches (Alais et al., 2010a), and that such a model can be located early in visual processing (Tong and Engel, 2001; Lee et al., 2005, 2007). Indeed, the sufficiency of the early process to produce switches may explain the finding in several reports that while removing attention slows the rate of rivalry alternations, the alternations continue to occur in the near-absence of attention (Paffen et al., 2006; Pastukhov and Braun, 2007; Paffen and Hooge, 2011). In addition, this suggestion is in line with a very recent report by Knapen et al. (2011), who showed that frontal activation related to perceptual alternations was more likely reflecting a response to these alternations rather than being their cause.

Human frontal cortex has been implicated as the site responsible for maintaining conscious representations of the visual world (Crick and Koch, 1995; Rees, 2001). From these observations, it could be hypothesized that prefrontal cortex is involved in selecting one of two competing images during binocular rivalry. Nonetheless, it is still possible that a similar pattern of activity would be seen in prefrontal cortex without endogenous attention, simply as a consequence of maintaining a conscious representation of the current winner of the early reciprocal inhibition process. Indeed, this would be consistent with the results of Windmann et al. (2006) who found that the ability to hold a percept dominant in perception was unaffected in patients with prefrontal lesions (although this study did not use binocular rivalry displays).

In sum, binocular rivalry may be considerably modulated by voluntary attention, but it is not under complete attentional control. We propose that there are two important reasons for this. First, an adapting reciprocal inhibition arrangement between early monocular channels is sufficient to trigger switches in perceptual dominance independently of a high-level "selection" mechanism. Second, the contrast enhancing effect of top-down attentional selection is counteracted over time by a response attenuating effect of neural adaptation which weakens the response to the dominant image. Both of these processes can be thought of as reciprocal and therefore self-balancing processes, with the consequence that complete attentional control over binocular rivalry could never be realized.

Blake, R., and Logothetis, N. K. (2002). Visual competition. Nat. Rev. Neurosci. 3, 13-21.

Blake, R., and Wilson, H. (2011). Binocular vision. Vision Res. 51, 754-770.

Brascamp, J. W., van Ee, R., Noest, A. J., Jacobs, R. H., and van den Berg, A. V. (2006). The time course of binocular rivalry reveals a fundamental role of noise. J. Vis. 6, 1244-1256.

Breese, B. B. (1899). On inhibition. Psychol. Monogr. 3, 1-15.

Britz, J., Landis, T., and Michel, C. M. (2009). Right parietal brain activity precedes perceptual alternation of bistable stimuli. Cereb. Cortex 19, 55-65.
Bundesen, C., and Habekost, T. (2008). Principles of Visual Attention: Linking Mind and Brain. Oxford: Oxford University Press.

Carmel, D., Walsh, V., Lavie, N., and Rees, G. (2010). Right parietal TMS shortens dominance durations in binocular rivalry. Curr. Biol. 20, R799-R800.

Carrasco, M., Ling, S., and Read, S. (2004). Attention alters appearance. Nat. Neurosci. 7, 308-313.

Chong, S. C., and Blake, R. (2006). Exogenous attention and endogenous attention influence initial dominance in binocular rivalry. Vision Res. 46, 1794-1803. 
Chong, S. C., Tadin, D., and Blake, R. (2005). Endogenous attention prolongs dominance durations in binocular rivalry. J. Vis. 5, 1004-1012.

Corbetta, M., and Shulman, G. L. (2002). Control of goal-directed and stimulus-driven attention in the brain. Nat. Rev. Neurosci. 3, 201-215.

Cosmelli, D., David, O., Lachaux, J.-P., Martinerie, J., Garnero, L., Renault, B., and Varela, F. (2004). Waves of consciousness: ongoing cortical patterns during binocular rivalry. $\mathrm{Neu}$ roimage 23, 128-140.

Crick, F., and Koch, C. (1995). Are we aware of neural activity in primary visual cortex? Nature 375, 121-123.

Desimone, R., and Duncan, J. (1995). Neural mechanisms of selective visual attention. Annu. Rev. Neurosci. 18, 193-222.

Duncan, J. (2001). An adaptive coding model of neural function in prefrontal cortex. Nat. Rev. Neurosci. 2, 820-829.

Fang, F., and He, S. (2005). Cortical responses to invisible objects in the human dorsal and ventral pathways. Nat. Neurosci. 8, 1380-1385.

George, R. W. (1936). The significance of the fluctuation experienced in observing ambiguous figures in binocular rivalry. J. Gen. Psychol. 15, 39-61.

Georgeson, M. A., and Meese, T. S. (1997). Perception of stationary plaids: the role of spatial filters in edge analysis. Vision Res. 37, 3255-3271.

Grindley, G. C., and Townsend, V. (1965). Binocular masking induced by a moving object. Q. J. Exp. Psychol. 17, 97-109.

Hancock, S., and Andrews, T. J. (2007). The role of voluntary and involuntary attention in selecting perceptual dominance during binocular rivalry. Perception 36, 288-298.

Haynes, J.-D., Deichmann, R., and Rees, G. (2005). Eye-specific effects of binocular rivalry in the human lateral geniculate nucleus. Nature 438, 496-499.

Helmholtz, H. V. (1925). Treatise on Physiological Optics. New York: Dover.

Hering, K. E. (1964). Outlines of a Theory of the Light Sense (L. M. Hurvich and D. Jameson, Trans.). Cambridge: Harvard University Press.

Jiang, Y., Costello, P., Fang, F., Huang, M., and He, S. (2006). A gender- and sexual orientation-dependent spatial attentional effect of invisible images. Proc. Natl. Acad. Sci. U.S.A. 103, 17048-17052.
Jiang, Y., and He, S. (2006). Cortical responses to invisible faces: dissociating subsystems for facialinformation processing. Curr. Biol. 16, 2023-2029.

Kamphuisen, A. P., Bauer, M., and van Ee, R. (2008). No evidence for widespread synchronized networks in binocular rivalry: MEG frequency tagging entrains primarily early visual cortex. J. Vis. 4.1-8.

Kanai, R., Bahrami, B., and Rees, G. (2010). Human parietal cortex structure predicts individual differences in perceptual rivalry. Curr. Biol. 20, 1626-1630.

Kanai, R., Carmel, D., Bahrami, B., and Rees, G. (2011). Structural and functional fractionation of right superior parietal cortex in bistable perception. Curr. Biol. 21, R106-R107.

Kastner, S., and Ungerleider, L. G. (2000). Mechanisms of visual attention in the human cortex. Annu. Rev. Neurosci. 23, 315-341.

Knapen, T., Brascamp, J., Pearson, J., van Ee, R., and Blake, R. (2011). The role of frontal and parietal areas in bistable perception. J. Neurosci. 31, 10293-10301.

Kovács, I., Papathomas, T. V., Yang, M., and Fehér, A. (1996). When the brain changes its mind: interocular grouping during binocular rivalry. Proc. Natl. Acad. Sci. U.S.A. 93, 15508-15511.

Lack, L. C. (1978). Selective Attention and the Control of Binocular Rivalry. The Hague: Mouton.

Lee, S.-H., Blake, R., and Heeger, D. J. (2005). Traveling waves of activity in primary visual cortex during binocular rivalry. Nat. Neurosci. 8, 22-23.

Lee, S.-H., Blake, R., and Heeger, D. J. (2007). Hierarchy of cortical responses underlying binocular rivalry. Nat. Neurosci. 10, 1048-1054.

Leopold, D. A., and Logothetis, N. K. (1999). Multistable phenomena: changing views in perception. Trends Cogn. Sci. (Regul. Ed.) 3, 254-264.

Levelt, W. J. M. (1965). On Binocular Rivalry. Institute for Perception RVO-TNO, Soesterberg.

Liu, L., Tyler, C. W., and Schor, C. M. (1992). Failure of rivalry at low contrast: evidence of a suprathreshold binocular summation process. Vision Res. 32, 1471-1479.

Logothetis, N. K., Leopold, D. A., and Sheinberg, D. L. (1996). What is rivalling during binocular rivalry? Nature 380, 621-624.

Lumer, E. D., Friston, K., and Rees, G. (1998). Neural correlates of perceptual rivalry in the human brain. Science 280, 1930-1934.
Lumer, E. D., and Rees, G. (1999). Covariation of activity in visual and prefrontal cortex associated with subjective visual perception. Proc. Natl. Acad. Sci. U.S.A. 96, 1669-1673.

McDougall, W. (1903). The physiological factors of the attention process. Mind 12, 473-488.

Meng, M., and Tong, F. (2004). Can attention selectively bias bistable perception? Differences between binocular rivalry and ambiguous figures. J. Vis. 4, 539-551.

Meredith, G. M., and Meredith, C. G. (1962). Effect of instructional conditions on rate of binocular rivalry. Percept. Mot. Skills 15, 655-664.

Miller, E. K., and Cohen, J. D. (2001). An integrative theory of prefrontal cortex function. Annu. Rev. Neurosci. 24 167-202.

Miller, S. M., Liu, G. B., Ngo, T. T., Hooper, G., Riek, S., Carson, R. G., and Pettigrew, J. D. (2000). Interhemispheric switching mediates perceptual rivalry. Curr. Biol. 10, 383-392.

Mitchell, J. F., Stoner, G. R., and Reynolds, J. H. (2004). Objectbased attention determines dominance in binocular rivalry. Nature 429, 410-413.

Morris, J. S., Ohman, A., and Dolan, R. J. (1999). A subcortical pathway to the right amygdala mediating "unseen" fear. Proc. Natl. Acad. Sci. U.S.A. 96, 1680-1685.

Nguyen, V. A., Freeman, A. W., and Alais, D. (2003). Increasing depth of binocular rivalry suppression along two visual pathways. Vision Res. 43, 2003-2008.

Ooi, T. L., and He, Z. J. (1999). Binocular rivalry and visual awareness: the role of attention. Perception 28, 551-574.

Paffen, C. L. E., Alais, D., and Verstraten, F. A. J. (2006). Attention speeds binocular rivalry. Psychol. Sci. 17, 752-756.

Paffen, C. L. E., Hooge, I., Benjamins, J. S., and Hogendoorn, H. (2011). A search asymmetry for interocular conflict. Atten. Percept. Psychophys. 73, 1042-1053.

Paffen, C. L. E., and Hooge, I. T. (2011). The effect of set size on the dynamics of binocular rivalry. Seeing Perceiving 24, 19-35.

Paffen, C. L. E., and Van der Stigchel, S. (2010). Shifting spatial attention makes you flip: exogenous visual attention triggers perceptual alternations during binocular rivalry. Atten. Percept. Psychophys. 72, 1237-1243.

Pashler, H., Dobkins, K., and Huang, L. (2004). Is contrast just another feature for visual selective attention? Vision Res. 44, 1403-1410.

Pastukhov, A., and Braun, J. (2007). Perceptual reversals need no prompting by attention. J. Vis. 7, 1-17.

Polonsky, A., Blake, R., Braun, J., and Heeger, D. J. (2000). Neural activity in human primary visual cortex correlates with perception during binocular rivalry. Nat. Neurosci. 3, 1153-1159.

Posner, M. I., and Dehaene, S. (1994). Attentional networks. Trends $\mathrm{Neu}$ rosci. 17, 75-79.

Rees, G. (2001). Neuroimaging of visual awareness in patients and normal subjects. Curr. Opin. Neurobiol. 11, 150-156.

Reisberg, D., and O'Shaughnessy, M. (1984). Diverting subjects' concentration slows figural reversals. Perception 13, 461-468.

Reynolds, J. H., and Chelazzi, L. (2004). Attentional modulation of visual processing. Annu. Rev. Neurosci. 27, 611-647.

Solomon, J. A., John, A., and Morgan, M. J. (2006). Monocular texture segmentation and proto-rivalry. Vision Res. 46, 1488-1492.

Srinivasan, R., Russell, D. P., Edelman, G. M., and Tononi, G. (1999) Increased synchronization of neuromagnetic responses during conscious perception. J. Neurosci. 19, 5435-5448.

Sterzer, P., and Kleinschmidt, A. (2007). A neural basis for inference in perceptual ambiguity. Proc. Natl. Acad. Sci. U.S.A. 104, 323-328.

Sterzer, P., and Rees, G. (2008). A neural basis for percept stabilization in binocular rivalry. J. Cogn. Neurosci. 20, 389-399. [Research Support, Non-U.S. Gov't].

Tong, F., and Engel, S. A. (2001). Interocular rivalry revealed in the human cortical blindspot representation. Nature 411, 195-199.

Tong, F., Meng, M., and Blake, R. (2006). Neural bases of binocular rivalry. Trends Cogn. Sci. 10, 502-511.

Tong, F., Nakayama, K., Vaughan, J. T., and Kanwisher, N. (1998). Binocular rivalry and visual awareness in human extrastriate cortex. Neuron 21, 753-759.

Tsuchiya, N., and Koch, C. (2005). Continuous flash suppression reduces negative afterimages. Nat. Neurosci. 8, 1096-1101.

van Dam, L. C. J., and van Ee, R. (2006a). The role of saccades in exerting voluntary control in perceptual and binocular rivalry. Vision Res. 46, 787-799. 
van Dam, L. C. J., and van Ee, R. (2006b). Retinal image shifts, but not eye movements per se, cause alternations in awareness during binocular rivalry. J. Vis. 6, 1172-1179.

van Ee, R., van Boxtel, J. J. A., Parker, A. L., and Alais, D. (2009). Multisensory congruency as a mechanism for attentional control over perceptual selection. J. Neurosci. 29, 11641-11649.

van Ee, R., van Dam, L. C. J., and Brouwer, G. J. (2005). Voluntary control and the dynamics of perceptual bi-stability. Vision Res. 45, 41-55.

Walker, P., and Powell, D. J. (1979). The sensitivity of binocular rivalry to changes in the nondominant stimulus. Vision Res. 19, 247-249.

Washburn, M. F., and Gillette, A. (1933). Studies from the psychological laboratorium of Vassar College; LXII. Motor factors in voluntary control of cube perspective fluctuations and retinal rivalry. Am. J. Psychol. 45, 315-319.

Weiskrantz, L. (1997). Consciousness Lost and Found: A Neuropsychological Exploration. Oxford: Oxford University Press.

Wheatstone, C. (1838). Contributions of the physiology of vision. Part the first. On some remarkable, and hitherto unobserved, phenomena of binocular vision. Philos. Trans. $R$. Soc. London 128, 371-394.

Wilson, H. R. (2003). Computational evidence for a rivalry hierarchy in vision. Proc. Natl. Acad. Sci. U.S.A. 100, 14499-14503.

Wilson, H. R., Blake, R., and Lee, S.H. (2001). Dynamics of travelling waves in visual perception. Nature 412, 907-910.

Windmann, S., Wehrmann, M., Calabrese, P., and Güntürkün, O. (2006). Role of the prefrontal cortex in attentional control over bistable vision. J. Cogn. Neurosci. 18, 456-471.
Wolfe, J. M. (1983). Influence of spatial frequency, luminance, and duration on binocular rivalry and abnormal fusion of briefly presented dichoptic stimuli. Perception 12, 447-456.

Wolfe, J. M., and Franzel, S. L. (1988). Binocularity and visual search. Percept. Psychophys. 44, 81-93.

Wright, R. D., and Ward, L. M. (2008). Orienting of Attention. Oxford: Oxford University Press.

Wunderlich, K., Schneider, K. A., and Kastner, S. (2005). Neural correlates of binocular rivalry in the human lateral geniculate nucleus. $\mathrm{Nat}$. $\mathrm{Neu}$ rosci. 8, 1595-1602.

Zanto, T. P., Rubens, M. T., Thangavel, A., and Gazzaley, A. (2011). Causal role of the prefrontal cortex in topdown modulation of visual processing and working memory. Nat. Neurosci. 14, 656-661.

Zaretskaya, N., Thielscher, A., Logothetis, N. K., and Bartels, A. (2010). Disrupting parietal function prolongs dominance durations in binocular rivalry. Curr. Biol. 20, 2106-2111.

Conflict of Interest Statement: The authors declare that the research was conducted in the absence of any commercial or financial relationships that could be construed as a potential conflict of interest.

Received: 21 July 2011; paper pending published: 10 August 2011; accepted: 08 September 2011; published online: 27 September 2011.

Citation: Paffen CLE and Alais D (2011) Attentional modulation of binocular rivalry. Front. Hum. Neurosci. 5:105. doi: 10.3389/fnhum.2011.00105

Copyright () 2011 Paffen and Alais. This is an open-access article subject to a nonexclusive license between the authors and Frontiers Media SA, which permits use, distribution and reproduction in other forums, provided the original authors and source are credited and other Frontiers conditions are complied with. 